Check for updates

Cite this: RSC Adv., 2017, 7, 37659

\title{
A novel and environmentally friendly bioprocess for separation and partial purification of polysaccharides from Cordyceps sinensis mycelia by an aqueous two-phase system $\dagger$
}

\author{
Zhigang Li, ${ }^{\text {ab }}$ Ailin Chen, ${ }^{a}$ Zhencheng Li, ${ }^{a}$ Man Qu, ${ }^{a}$ Huayong Chen, ${ }^{\text {ab }}$ Bo Yang ${ }^{\star a b}$ \\ and Yonghua Wang (D) *c
}

Separation and partial purification of polysaccharides from fungi or mushrooms are difficult and have become the main bottleneck in the development of a commercially viable process. In this study, an aqueous two-phase system (ATPS) composed of short chain alcohols and salts was developed for the separation and partial purification of polysaccharides and other bioactive constituents from Cordyceps sinensis (CS). The results showed that ATPS not only purified CS polysaccharides (CSPS) with the removal of $99.1 \%$ cell debris, $80 \%$ proteins, $90.7 \%$ pigments, and $61.3 \%$ nucleic acids, but also simultaneously recovered CSPS, adenosine, and mannitol up to $96.6 \%, 100 \%$, and $88.4 \%$, respectively. Compared with traditional purification methods, the coupling of ethanol/ $\mathrm{Na}_{2} \mathrm{CO}_{3}$ ATPS with ultrafiltration decreased the protein, nucleic acid, and pigment contents in the purified CSPS by $344 \%, 192 \%$, and $290 \%$, respectively. Therefore, this environmentally friendly method could have potential applications in large-scale separation of multiple bioactive constituents from fungi in the future.

Received 12th May 2017
Accepted 11th July 2017
DOI: 10.1039/c7ra05360f
rsc.li/rsc-advances

\section{Introduction}

Polysaccharides have been widely applied in the food and pharmaceutical industries due to their interesting biological activities and low toxicity. ${ }^{\mathbf{1}}$ Recently, fungal-derived polysaccharides isolated from fruiting body and cultured mycelium have drawn significant attention due to their various pharmacological properties such as antioxidant, immunomodulatory, hepatoprotective, and anti-inflammatory properties as well as inhibitory properties towards tumor growth. ${ }^{2}$ However, the high cost of polysaccharide-based products (most of which are based on intracellular polysaccharides of fungi) has restricted their commercial applications because of the difficult separation and purification of polysaccharides from fungi. Therefore, an inexpensive, efficient, and scalable downstream process to obtain this product has always been the focus of research and development. $^{3}$

In the past 20 years, many techniques, such as alkaline extraction, ethanol precipitation, fractional precipitation, ion-

${ }^{a}$ School of Bioscience and Bioengineering, South China University of Technology, Guangzhou 510006, China. E-mail: yangbo@scut.edu.cn

${ }^{b}$ Guangdong Provincial Key Laboratory of Fermentation and Enzyme Engineering, China

${ }^{c}$ School of Light Industry and Food Sciences, South China University of Technology, Guangzhou 510641, China

$\dagger$ Electronic supplementary information (ESI) available. See DOI: 10.1039/c7ra05360f exchange chromatography, gel filtration, and affinity chromatography, have been developed to separate and purify polysaccharides from fungi or mushrooms. ${ }^{2}$ The conventional method to separate polysaccharides from fungi involves centrifugation to remove bacterial cells, precipitation of the supernatant with alcohol, and ultrafiltration to remove the small molecules. However, the cell debris and biomacromolecular impurities (proteins, pigments, and nucleic acids) also precipitated with the intracellular polysaccharides and could not be removed via ultrafiltration due to the similar molecular weights of the biomacromolecular impurities and polysaccharides. To meet the high requirements of purity of the polysaccharides, complicated processes, such as membrane filtration or high-speed centrifugation for the removal of cell debris, macroporous resin adsorption for decoloration, and Sevag method for the removal of proteins, were necessary to remove biomacromolecular impurities. ${ }^{4-6}$ As a result, the cost of downstream processing constitute a very high portion of the total production costs due to the massive loss of polysaccharides and complicated processes. More importantly, most fungi usually contain a variety of other bioactive substances, which are usually lost during the purification process. This will not only lead to substantial economic losses, but also increase environmental pollution. To overcome the drawbacks of the existing downstream techniques, a novel method based on an aqueous two-phase system (ATPS) consisting of short chain alcohols and salts was developed. 
A conventional ATPS consisting of a polymer/salt system, ionic liquid/salt system, or polymer/polymer system has been widely applied in the separation of biomacromolecules because of its mild conditions, high capacity, and easy scale-up. ${ }^{2,7,8}$ However, these ATPSs have been rarely used for commercial applications due to the high cost of the extractant, potential toxicity of the ionic liquid, and the difficulty in the recovery of extracted molecules from the polymer or ionic liquid phase. In fact, short chain alcohols and salts are able to form an ATPS at appropriate concentrations. ${ }^{9}$ This ATPS has a number of advantages, such as high extraction efficiency, low cost of the extractants, and ease of recovery of the extractants, over the traditional systems. ${ }^{\mathbf{1 0}}$ In our previous study, this ATPS has successfully been used for the recovery of bio-based chemicals such as butanol, 1,3-propanediol, and 2,3-butanediol. ${ }^{\mathbf{1 0 - 1 3}}$ However, to the best of our knowledge, there have been no reports on the use of this system in the separation of intracellular polysaccharides from fungi or mushrooms.

Cordyceps sinensis (CS), one of the most valued traditional Chinese medicines, ${ }^{\mathbf{1 4}, 15}$ has drawn significant attention due to its multiple pharmacological functions such as immunoregulation, anti-oxidant properties, inhibition of tumor growth, and prevention of cardiac arrhythmias. ${ }^{16,17}$ Similar to other medicinal fungi, CS mycelium also contains a variety of bioactive substances. For example, CS polysaccharides (CSPS), adenosine, and mannitol are the major contributors towards the inhibition of tumor growth, control of blood flow, and anti-lipid peroxidation of CS, respectively. Recently, CSPS have received particular interest due to their high activities such as antioxidant, antitumor, antivirus, and so on. ${ }^{18-22}$ However, the commercial application of CSPS is limited by their complicated operations, high operating costs to purify the CSPS, as well as substantial loss of other bioactive substances. Herein, a bioprocess has been described for the separation and partial purification of CSPS from cultured Cordyceps using ATPSs consisting of short chain alcohols and salts. In this ATPS, the CSPS, cell debris, and the macromolecular impurities (pigments, proteins, and nucleic acids) were partitioned, respectively, into the bottom, intermediate solid, and top phases. As a result, a series of complex separation process, such as the removal of cell debris, decoloration, and deproteinization, can be economically integrated into one step. More importantly, in this system, the other two main bioactive micromolecules adenosine and mannitol could be simultaneously separated because they could be extracted into various phases and easily separated from the macromolecules in the corresponding phases via ultrafiltration due to the obvious differences in molecular weight. Therefore, herein, we provided a simple, costeffective, and environmentally friendly method for the separation and purification of multiple bioactive constituents from fungi.

\section{Materials and methods}

\subsection{Materials}

All experiments were performed using deionized water. Adenosine and mannitol standards were purchased from Sigma
Chemical Co. Cultured CS mycelia were obtained from Infinitus (China) Co. Ltd in the Guangdong province. BCA kit was purchased from Nanjing KeyGen Biotech. Co. Ltd. All the other chemicals were of analytical grade.

\subsection{Partition behavior of adenosine, mannitol, and CSPS in ATPS}

Cultured CS mycelia was dissolved in 20 volumes of water (solvent to sample ratio, v/w) at room temperature. The CS mycelia was disrupted via ultrasound $(250 \mathrm{~W})$ for $30 \mathrm{~min}$ and then held for $3 \mathrm{~h}$ at $80{ }^{\circ} \mathrm{C}$. The resulting CS mycelia lysate was centrifuged at $10000 \mathrm{rpm}$ for $10 \mathrm{~min}$. The partition experiments were carried out by adding alcohol and solid salt to the supernatant to form ATPSs consisting of $15-30 \%(\mathrm{w} / \mathrm{w})$ salt and $20 \%$ (w/w) ethanol. The mixture was vortexed for $1 \mathrm{~min}$ and then centrifuged at $3000 \mathrm{rpm}$ for $1 \mathrm{~min}$. This separation process was tested with different salts and ethanol using separate samples of the supernatant. The partition coefficient $K$ of adenosine, mannitol, and CSPS was defined as the ratio of the concentration of adenosine, mannitol, and CSPS in the top phase to that in the bottom phase, respectively. The recovery of adenosine ( $\left.Y_{\text {adenosine }}\right)$ was the ratio of adenosine partitioned in the top phase to the total amount of adenosine. It was determined via the partition coefficient of adenosine and the phase volume ratio ( $R$, volume ratio of the top phase to the bottom phase) via the following equation:

$$
Y_{\text {adenosine }}=\frac{R K_{\text {adenosine }}}{1+R K_{\text {adenosine }}} \times 100 \%
$$

The recovery of mannitol and CSPS ( $Y_{\text {mannitol }}$ and $\left.Y_{\mathrm{CSPS}}\right)$ was the ratio of mannitol and CSPS partitioned in the bottom phase to the total amount of mannitol and CSPS, respectively. It was determined via the partition coefficient of mannitol and CSPS and the volume ratio of the top phase to the bottom phase using the following equation:

$$
\begin{gathered}
Y_{\mathrm{CSPS}}=\frac{1}{1+R K_{\mathrm{CSPS}}} \times 100 \% \\
Y_{\text {mannitol }}=\frac{1}{1+R K_{\text {mannitol }}} \times 100 \%
\end{gathered}
$$

The selective coefficient of adenosine to mannitol and CSPS was calculated as the ratio of the partition coefficient of adenosine to that of mannitol and CSPS, respectively.

\subsection{Effects of $\mathrm{Na}_{2} \mathrm{CO}_{3}$ and ethanol on the partition of adenosine, mannitol, and CSPS in ATPS}

The effects of $\mathrm{Na}_{2} \mathrm{CO}_{3}$ and ethanol were investigated by adding solid $\mathrm{Na}_{2} \mathrm{CO}_{3}$ and ethanol to the supernatant of CS mycelia lysate to form ATPSs consisting of $9-27 \%(\mathrm{w} / \mathrm{w})$ ethanol and $7-20 \%(\mathrm{w} / \mathrm{w}) \mathrm{Na}_{2} \mathrm{CO}_{3}$. The mixtures were vortexed for $1 \mathrm{~min}$ and then centrifuged at $3000 \mathrm{rpm}$ for $1 \mathrm{~min}$. The partition coefficients $K$ and removal ratios of the cell debris, nucleic acid, pigment, and protein were then examined. The partition 
coefficients $K$ of nucleic acid, pigment, and protein were defined as the ratio of the concentration of nucleic acid, pigment, and protein in the top phase to that in the bottom phase, respectively. The removal ratios of cell debris were expressed as the ratio of the cell debris partitioned in the middle phase to the total amount in the sample. The removal ratios of nucleic acid, pigment, and protein were expressed as the ratio of nucleic acid, pigment, and protein partitioned in the middle and top phases to the total amounts in the sample, respectively. The selective coefficient of nucleic acid, pigment, and protein to CSPS was calculated as the ratio of the partition coefficient of nucleic acid, pigment, and protein to that of CSPS, respectively.

\subsection{Isolation and partial purification of the CSPS}

Herein, $50 \mathrm{~g}$ cultured CS mycelia was dissolved in $1000 \mathrm{~L}$ water at room temperature. The CS mycelia of the mixture was disrupted via ultrasound $(250 \mathrm{~W})$ for $30 \mathrm{~min}$ and then held for $3 \mathrm{~h}$ at $80{ }^{\circ} \mathrm{C}$. The solution was then centrifuged at $10000 \mathrm{rpm}$ for $10 \mathrm{~min}$. The CSPS of the supernatant was extracted via the ATPS consisting of $15 \%(\mathrm{w} / \mathrm{w})$ ethanol and $20 \%(\mathrm{w} / \mathrm{w}) \mathrm{Na}_{2} \mathrm{CO}_{3}$. As a control experiment, the supernatant was precipitated with four volumes of absolute ethanol at $4{ }^{\circ} \mathrm{C}$ for $12 \mathrm{~h}$ and then deproteinized via a Sevag method 8 times. Then, the bottom phase and the control were exhaustively dialyzed against water for $72 \mathrm{~h}$ and precipitated with four volumes of absolute ethanol at $4{ }^{\circ} \mathrm{C}$ for $12 \mathrm{~h}$, respectively. The precipitates were evaporated under vacuum and freeze-dried to obtain the CSPS.

\subsection{Analytical methods}

An HPLC system (Waters 1525) with a UV230+ detector and analytical software was used for the detection and analysis of the adenosine concentrations in the samples. The HPLC conditions were as follows: column, Hypersil Xbridge C18 $(4.6 \mathrm{~mm} \times 250 \mathrm{~mm}, 5 \mu \mathrm{m})$; mobile phase, a mixture of $2 \%$ tetrahydrofuran and $98 \%$ phosphate buffer $\left(0.66 \mathrm{~mol} \mathrm{~L}^{-1}\right.$, $\left.\mathrm{KH}_{2} \mathrm{PO}_{4}: \mathrm{Na}_{2} \mathrm{HPO}_{4}=2: 3\right)$; flow rate, $1.0 \mathrm{~mL} \mathrm{~min}{ }^{-1}$; UV detection at $260 \mathrm{~nm}$; and injection amount, $10 \mu \mathrm{L} .{ }^{23}$ Mannitol concentration was determined using a colorimetry method. ${ }^{19}$ The concentration of the CSPS was analyzed using a phenolsulfuric acid method. ${ }^{24}$ The purity of the CSPS was defined as the ratio of the CSPS weight in the sample to the total weight of the sample. The concentration of protein was determined via the BCA kit (Sangon, Shanghai, China) and the standard curve was made using different concentrations of the BSA standard protein. ${ }^{25}$ The nucleic acid concentration was determined using a nucleic acid analyzer. The pigment and cell debris concentration was spectrophotometrically determined at $430 \mathrm{~nm}$ and $650 \mathrm{~nm}$, respectively. ${ }^{26}$ The molecular weights of the CSPS purified by ATPS and traditional method were determined via high performance gel permeation chromatography (HPGPC) using an HPLC system with a refractive index detector and two chromatographic columns TSK-GEL G-5000PWXL column $(7.8 \mathrm{~mm} \times 300 \mathrm{~mm})$ and TSK-GEL G-3000PWXL column $(7.8 \mathrm{~mm} \times 300 \mathrm{~mm})$ in series. The column oven temperature was $35^{\circ} \mathrm{C}$. The mobile phase was phosphate buffer $(0.2 \mathrm{M}, \mathrm{pH}=6.0)$ and the flow rate was $0.6 \mathrm{~mL} \mathrm{~min}^{-1}$. Solutions of the samples and standards $\left(1 \mathrm{mg} \mathrm{mL} \mathrm{mL}^{-1}\right.$ ) were filtered through $0.45 \mu \mathrm{m}$ membranes, and the injection volume was $10 \mu \mathrm{L}$.

\section{Results and discussion}

\subsection{Partition of CSPS, adenosine, and mannitol in ATPSs}

Ethanol has been widely used in the extraction and purification of bioactive constituents from CS mycelia due to its high extraction ability for biological materials, low toxicity, and easy recovery. ${ }^{19,27}$ Thus, ethanol was chosen as an organic solvent for the ATPS used in the extraction and purification of bioactive constituents. Different ATPSs consisting of either one of the five different salts $\left(\mathrm{K}_{2} \mathrm{HPO}_{4}, \mathrm{Na}_{2} \mathrm{CO}_{3}, \mathrm{Na}_{2} \mathrm{SO}_{4}, \mathrm{~K}_{2} \mathrm{CO}_{3}\right.$, and $\left.\left(\mathrm{NH}_{4}\right)_{2} \mathrm{SO}_{4}\right)$ and ethanol were used to separate the CSPS, adenosine, and mannitol from CS mycelia, and the results are shown in Table 1. Although adenosine has great affinity to water, ${ }^{28}$ it is partitioned into the top (ethanol) phase with a high partition coefficient and recovery due to the salting-out effect $\left(R_{\text {adenosine }}>96 \%\right)$. On the contrary, CSPS and mannitol generally tend to get partitioned into the bottom phase. This is because mannitol and CSPS (contain sugar chain) possess very high polarity to be extracted to the top phase via the ATPS. In all the tested ATPSs, the high selective coefficients of adenosine to mannitol and CSPS could be obtained (above 50), indicating that most of adenosine could be separated from CSPS and mannitol by adjusting the extraction methods such as the use of multi-stage cross flow or countercurrent extraction. Although ethanol $/ \mathrm{K}_{2} \mathrm{CO}_{3}$ ATPS achieved the best extraction efficiency, the high dosage and cost of the salt as well as the difficulty in the recovery of the salt limited its large-scale application (Fig. S1 $\dagger$ ). Among these ATPSs, ethanol/ $/ \mathrm{Na}_{2} \mathrm{CO}_{3}$ ATPS not only effectively extracted the bioactive constituents from CS mycelia, but also was a more economical method at the commercial level due to its economic cost, requirement of lower dosage of salt, and lower energy consumption during the recycling of salt and ethanol $^{13}$ (Fig. S1 $\dagger$ ). Thus, the ATPS consisting of ethanol and $\mathrm{Na}_{2} \mathrm{CO}_{3}$ was chosen for subsequent experiments.

\subsection{The effects of ethanol and $\mathrm{Na}_{2} \mathrm{CO}_{3}$ concentrations on the partitioning of CSPS, adenosine, and mannitol in ATPS}

The effects of ethanol and $\mathrm{Na}_{2} \mathrm{CO}_{3}$ concentrations on the partitioning of CSPS are shown in Fig. 1. Most of the partition coefficients of CSPS were under 0.15, indicating that most CSPS could be effectively concentrated in the bottom (salt-rich)

Table 1 Effect of the type of salts on the partitions of adenosine, mannitol, and CSPS in different ATPSs

\begin{tabular}{|c|c|c|c|c|c|c|}
\hline & \multicolumn{2}{|c|}{ Adenosine } & \multicolumn{2}{|c|}{ CSPS } & \multicolumn{2}{|c|}{ Mannitol } \\
\hline & $K$ & $Y(\%)$ & $K$ & $Y(\%)$ & $K$ & $Y(\%)$ \\
\hline $\mathrm{K}_{2} \mathrm{CO}_{3}$ & 29.48 & 98.21 & 0.07 & 88.50 & 0.1 & 84.34 \\
\hline $\mathrm{K}_{2} \mathrm{HPO}_{4}$ & 22.63 & 96.12 & 0.19 & 82.78 & 0.11 & 89.25 \\
\hline $\mathrm{Na}_{2} \mathrm{SO}_{4}$ & 67.68 & 97.88 & 0.9 & 62.01 & 1.28 & 53.44 \\
\hline $\mathrm{Na}_{2} \mathrm{CO}_{3}$ & 49.36 & 98.21 & 0.16 & 84.92 & 0.25 & 78.28 \\
\hline$\left(\mathrm{NH}_{4}\right)_{2} \mathrm{SO}_{4}$ & 32.99 & 98.90 & 0.23 & 61.57 & 0.23 & 61.57 \\
\hline
\end{tabular}



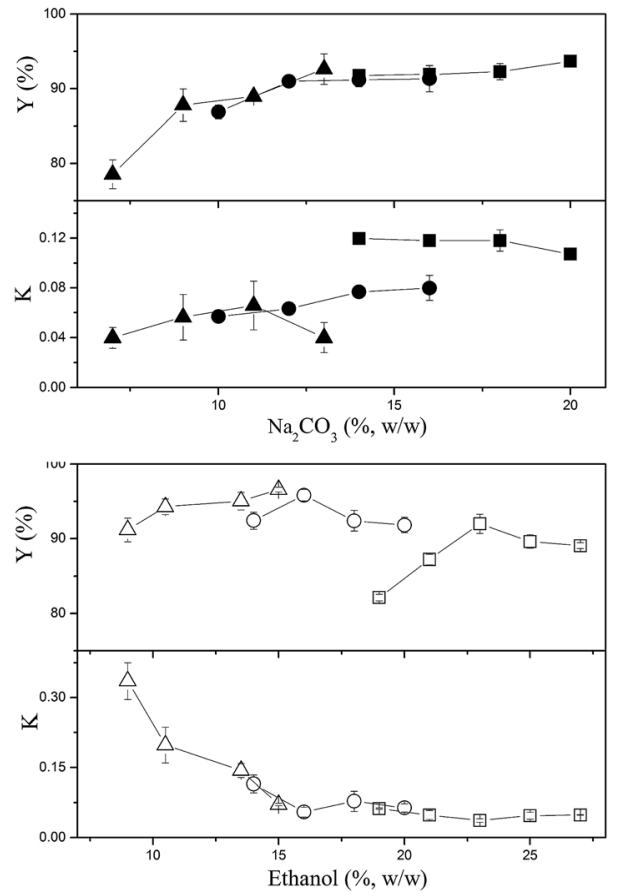

Fig. 1 Effects of $\mathrm{Na}_{2} \mathrm{CO}_{3}$ and ethanol concentrations on the partition coefficient and recovery of CSPS in the ethanol/ $\mathrm{Na}_{2} \mathrm{CO}_{3}$ ATPS. Top: Effects of $\mathrm{Na}_{2} \mathrm{CO}_{3}$ concentrations; bottom: effects of ethanol

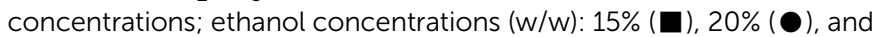
$25 \%(\boldsymbol{\Delta})$; and $\mathrm{Na}_{2} \mathrm{CO}_{3}$ concentrations (w/w): $10 \%(\square), 15 \%(O)$, and $20 \%(\Delta)$.

phase. In general, ethanol was more effective in influencing the partition coefficients of CSPS than $\mathrm{Na}_{2} \mathrm{CO}_{3}$. With the increase in ethanol concentration, the partition coefficients of CSPS dramatically decreased, but the effect of salt concentration was not obvious. Furthermore, the change in the recovery of CSPS was more complicated than that in the partition coefficient of the CSPS. This is because the change in the recoveries was an integrated result of the partition coefficient of CSPS and the phase volume ratio. The phase volume ratio increased when the ethanol concentration was increased, but decreased when the $\mathrm{Na}_{2} \mathrm{CO}_{3}$ concentration was increased. Partitioning of adenosine is shown in Fig. 2. When the concentrations of ethanol and $\mathrm{Na}_{2} \mathrm{CO}_{3}$ were increased, the partition coefficients of adenosine were enhanced. In general, a slower increase in the partition coefficient of adenosine was observed. The effects of ethanol and $\mathrm{Na}_{2} \mathrm{CO}_{3}$ concentrations were observed upon increasing the concentration of ethanol rather than that of $\mathrm{Na}_{2} \mathrm{CO}_{3}$. This is because the ability of the salt to capture water molecules was stronger than that of ethanol, resulting in a greater effect of the salt on the partitioning behavior of adenosine. Similar high influences of salts and sugars on the solubility and extraction efficiencies of some small molecules have also been reported in some previous studies. ${ }^{30-32}$

At relatively high concentrations of ethanol and $\mathrm{Na}_{2} \mathrm{CO}_{3}$, the partition coefficients and recoveries of adenosine reached infinity and $100 \%$, respectively. This indicated that all of the adenosine was enriched in the top phase. When the system was composed of $15 \%(w / w)$ ethanol and $20 \%(w / w) \mathrm{Na}_{2} \mathrm{CO}_{3}$, nearly
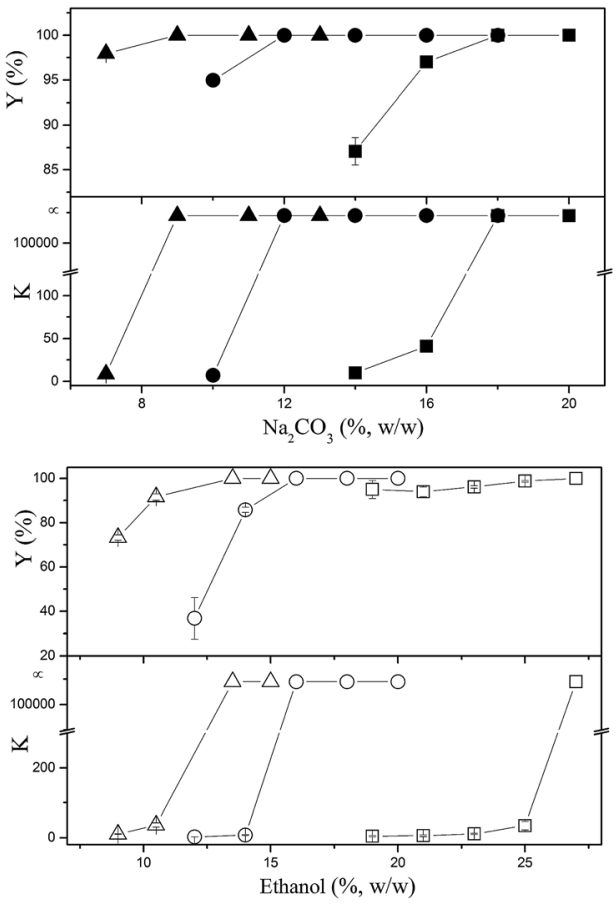

Fig. 2 Effects of $\mathrm{Na}_{2} \mathrm{CO}_{3}$ and ethanol concentrations on the partition coefficient and recovery of adenosine in the ethanol/ $/ \mathrm{Na}_{2} \mathrm{CO}_{3}$ ATPS. Top: Effects of $\mathrm{Na}_{2} \mathrm{CO}_{3}$ concentrations; bottom: effects of ethanol concentrations; ethanol concentrations (w/w): 15\% ( $\boldsymbol{\square}), 20 \%(\bullet)$, and $25 \%(\boldsymbol{\Delta}) ; \mathrm{Na}_{2} \mathrm{CO}_{3}$ concentrations (w/w): $10 \%(\square), 15 \%(O)$, and 20\% $(\triangle)$

$100 \%$ of adenosine was partitioned in the top phase, and the recovery of CSPS in the bottom phase also reached $96.6 \%$. This system is useful for the development of a commercially viable process by which two main bioactive constituents can be separated via a single extraction step rather than multiple steps or a continuous extraction.

In general, the effects of ethanol and $\mathrm{Na}_{2} \mathrm{CO}_{3}$ concentrations on the partition coefficients and recoveries of mannitol were similar to those of CSPS except for the fact that higher partition coefficients and lower recoveries of mannitol were observed (Fig. 3). This might be due to the weaker hydrophilicity of mannitol as compared to that of the CSPS. When the system was composed of $15 \%(\mathrm{w} / \mathrm{w})$ ethanol and $20 \%(\mathrm{w} / \mathrm{w}) \mathrm{Na}_{2} \mathrm{CO}_{3}, 88.4 \%$ of mannitol was concentrated in the bottom phase. It is helpful that the vast majority of mannitol was enriched in the bottom phase as it would enable easy separation from CSPS via ultrafiltration, a step that is necessary for the purification of CSPS.

Considering both the high recovery of the main bioactive constituent and the suitable phase volume ratio $(R)$, ATPS composed of $15 \%(\mathrm{w} / \mathrm{w})$ ethanol and $20 \%(\mathrm{w} / \mathrm{w}) \mathrm{Na}_{2} \mathrm{CO}_{3}$ was chosen as the optimum system for investigating the isolation and purification of the CSPS from CS mycelia.

\subsection{Removal of cell debris and biomacromolecular impurities}

When ATPS was directly used to separate the bioactive constituents from the CS mycelia lysate, a third phase or an 

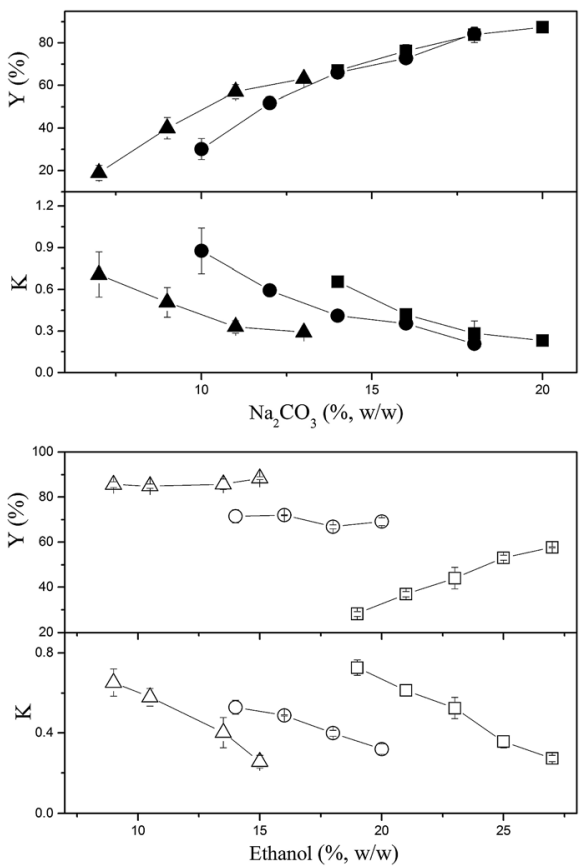

Fig. 3 Effects of $\mathrm{Na}_{2} \mathrm{CO}_{3}$ and ethanol concentrations on the partition coefficient and recovery of mannitol in the ethanol/ $\mathrm{Na}_{2} \mathrm{CO}_{3}$ ATPS. Top: Effects of $\mathrm{Na}_{2} \mathrm{CO}_{3}$ concentrations; bottom: effects of ethanol concentrations; ethanol concentrations (w/w): 15\% (ם), 20\% (-), and $25 \%(\boldsymbol{\Delta}) ; \mathrm{Na}_{2} \mathrm{CO}_{3}$ concentrations (w/w): $10 \%(\square), 15 \%(O)$, and $20 \%$ $(\triangle)$.

interphase was formed by the accumulation of cell debris at the interface between the top and bottom phases. As shown in Fig. 4, the removal ratio of cell debris reached $99.1 \%$, indicating that almost all the cell debris could be removed. In the downstream processing of intracellular macromolecules, separation of soluble macromolecules from cell debris was the main challenge because the cell debris was too small to be effectively removed via industrial centrifugation or microfiltration. Usually, some additional steps, such as flocculation and chromatographic purification, are necessary to improve the removal efficiency of cell debris. While using an ATPS, this step can be economically achieved at the same time. Furthermore, an ATPS could not only efficiently separate

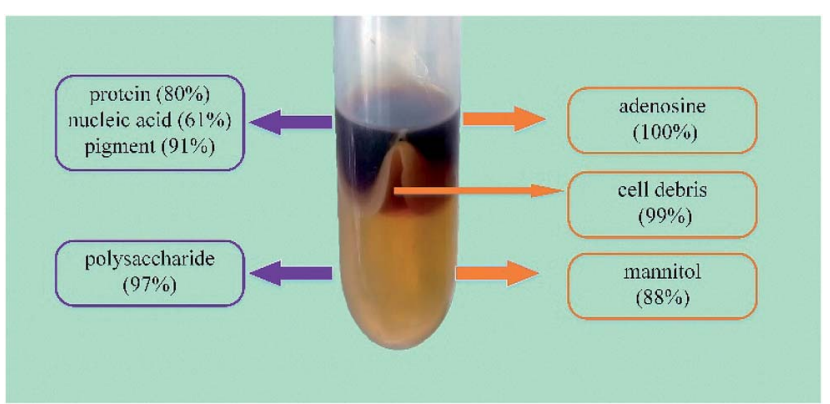

Fig. 4 Partitions of the main components of CS mycelia via the ethanol/ $/ \mathrm{Na}_{2} \mathrm{CO}_{3}$ ATPS. active small molecules and cell debris from the CS mycelia lysate, but also remove biomacromolecular impurities in the purification process for CSPS as the vast majority of pigments, nucleic acids, and proteins could be partitioned into the top phase. The partition coefficients of the main biomacromolecular impurities were above 3 , and the removal ratio of the pigments, proteins and nucleic acids reached $90.7 \%, 80.0 \%$, and $61.3 \%$, respectively. This is a good phenomenon because the removal of biomacromolecular impurities has been a bottleneck in the purification process for the CSPS. Rui et al. have isolated a macroporous resin (S-8) that can be used for the simultaneous decoloration and deproteinization of crude polysaccharides. The removal ratio of the pigment and protein reached $84.3 \%$ and $75.9 \%$, respectively. However, the removal ratio of the nucleic acid was not mentioned, and the recovery of the CSPS was only $84.7 \% .^{5}$ Herein, the ATPS could not only simultaneously remove the three main biomacromolecular impurities with higher efficiencies, but also dramatically increase the recovery of CSPS up to $96.6 \%$. Furthermore, adenosine in the top phase could be easily separated from the biomacromolecular impurities via ultrafiltration.

In general, the utilization of an ATPS can simplify the primary purification of the CSPS from the CS mycelia lysate. The down-stream processing of intracellular polysaccharides usually includes three main steps. The first step is the removal of cell debris mostly via membrane filtration and high-speed centrifugation. The second step is the recovery of the target constituent or removal of some primary impurities, such as alcohol precipitation for recovering CSPS, microwave-assisted extraction for recovering mannitol, and Sevag method for removing proteins. ${ }^{4,25,26}$ The last step is the final purification of the active constituents via column chromatography and freeze drying. While using an ATPS, the first two steps can be integrated into one step in an economical way.

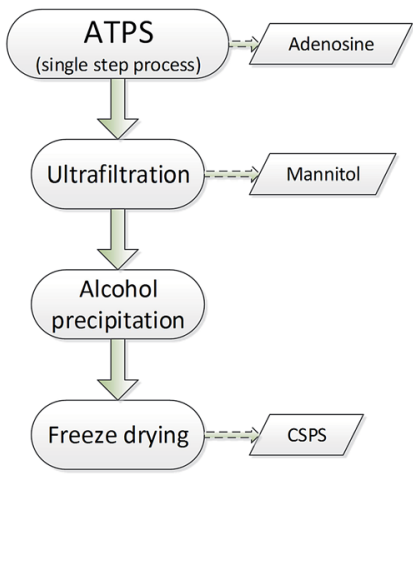

(A)

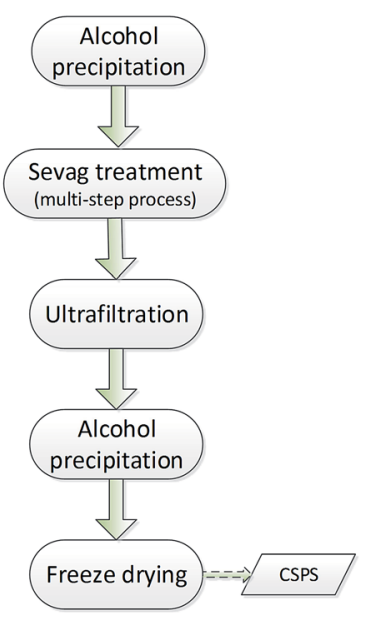

(B)
Fig. 5 Flow charts of the ATPS and traditional method. (A) Ethanol/ $\mathrm{Na}_{2} \mathrm{CO}_{3}$ ATPS and (B) traditional method. 
Table 2 Main components of partially purified CSPS via the ATPS and traditional method $^{a}$

\begin{tabular}{llllll}
\hline $\begin{array}{l}\text { Purification } \\
\text { method }\end{array}$ & CSPS $(\%)$ & $\begin{array}{l}\text { Protein } \\
(\%)\end{array}$ & $\begin{array}{l}\text { Nucleic } \\
\text { acid }(\%)\end{array}$ & $\mathrm{OD}_{650}$ & $\mathrm{OD}_{430}$ \\
\hline ATPS & 72.7 & 1.53 & 1.12 & 0.01 & 0.048 \\
Traditional & 67.6 & 5.26 & 2.15 & 0.035 & 0.139
\end{tabular}

method

${ }^{a} \mathrm{OD}_{650}$ : proportional to the cell debris content; $\mathrm{OD}_{430}$ : proportional to the pigment content.

\subsection{Isolation and partial purification of the CSPS}

The traditional processing for CSPS separation and purification usually includes multiple steps. ${ }^{29,33}$ As shown in Fig. 5B, CSPS were first precipitated from the CS mycelia lysate by ethanol. The precipitate was redissolved in water and treated many times with the Sevag reagent (1-butanol/chloroform $1: 4, \mathrm{v} / \mathrm{v}$ ) for the removal of proteins. Then, ultrafiltration was conventionally used for the removal of small molecular constituents. The partially purified CSPS were subsequently obtained via ethanol precipitation and then freeze-dried. In Fig. 5A, ATPS extraction instead of ethanol precipitation and Sevag treatment was used for the removal of microbial cell debris and biomacromolecular impurities. In this step, nearly $100 \%$ of adenosine could be simultaneously separated from mannitol and CSPS. Then, mannitol and CSPS were separated via ultrafiltration. Finally, the partial purified CSPS were obtained via the last two steps, similar to the traditional method.

Compared with the traditional method, the novel method based on ATPS can not only remove more impurities from the CSPS, but also simultaneously recover more bioactive substances. As shown in Table 2, the purity of CSPS purified by ATPS coupling with ultrafiltration was increased from $67.6 \%$ to $72.7 \%$, relative to that of the traditional method. The main increase in CSPS purity originated from two sources: (1) the removal ratio of proteins via the ATPS method was much higher than that obtained via the Sevag method. This is because the removal of the protein in the process of Sevag treatment is only through denaturing of proteins. However, under the extraction condition of ATPS, both the denatured and undenatured

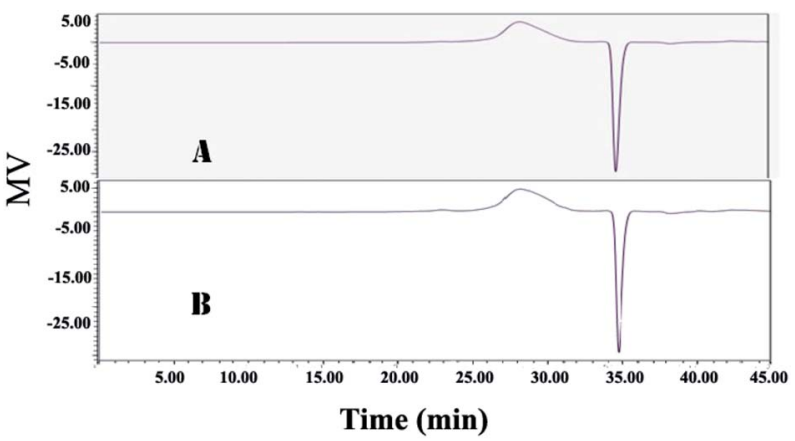

Fig. 6 HPGPC analysis of the CSPS purified by the ATPS and traditional method. (A) Traditional method and (B) ethanol/ $\mathrm{Na}_{2} \mathrm{CO}_{3}$ ATPS. protein were partitioned into the top phase or accumulated in the interphase. As a result, the protein content in the CSPS purified by the ATPS method was decreased by $344 \%$ relative to that of the traditional method. (2) In the novel method, the nucleic acids and pigments could be simultaneously removed, but in the process of Sevag treatment, almost no effect on the removal of these two impurities could be observed. Usually, some additional steps, such as $\mathrm{H}_{2} \mathrm{O}_{2}$ and chromatographic purification, were necessary to remove nucleic acids and pigments from the crude CSPS. Compared to those obtained via the traditional method, the nucleic acid and pigment contents in the CSPS purified via the ATPS method were dramatically decreased by $192 \%$ and $290 \%$, respectively. Furthermore, compared with the adverse impacts of the traditional method on environment and health, almost no toxic substances were used in the process of the novel method. Lower energy expenditure and separation cost of ATPS could also be obtained because the extractant dosage of ATPS was far less than the dosage of the Sevag reagent in the traditional method. As shown in Fig. 6, it was observed that there were no obvious changes in proportion and distribution of the only peak $\left(\mathrm{ca} .2 .57 \times 10^{6} \mathrm{KD}\right)$; this demonstrated that it was non-detectable. The novel method based on the ATPS could be a promising strategy for the degradation of CSPS produced during the treatment process; thus, majority of the CSPS can be retained without destroying its original molecular weights, whereas proteins, pigments, nucleic acids, and cell debris can be simultaneously removed from the CSPS. The abovementioned results showed that it was feasible to separate multiple bioactive constituents from the CS mycelia lysate using the ethanol/ $\mathrm{Na}_{2} \mathrm{CO}_{3}$ ATPS. The extraction process not only effectively extracted adenosine, mannitol, and CSPS, but also simultaneously removed cell debris, proteins, pigments, and nucleic acids. Further, this process is also easy to scale-up and has very low solvent/waste impact.

\section{Conclusion}

Ethanol/ $\mathrm{Na}_{2} \mathrm{CO}_{3}$ ATPS not only simultaneously recovered up to $100 \%$ of adenosine, $88.4 \%$ of mannitol, and $96.6 \%$ of CSPS, respectively, but also simultaneously removed cell debris, proteins, pigments, and nucleic acids from the CSPS. Furthermore, ATPS coupled with ultrafiltration was developed to purify the CSPS with high efficiency. Compared with those obtained via the traditional method, the protein, nucleic acid, and pigment contents in the purified CSPS via the ATPS method were also decreased by $344 \%, 192 \%$, and $290 \%$, respectively. This new strategy could be employed as a cost-effective, easy scale-up, and environmentally friendly method to separate and purify multiple bioactive constituents form fungi. It may also be used for further pharmacological and structural research of the CSPS.

\section{Acknowledgements}

This work was supported by the National Natural Science Foundation of China (Grant No. 2017MS078), the National High-tech R\&D Program of China (863 Program, Grant No. 
2014AA093601), and the Science and Technology Program of Guangzhou, China (Grant No. 201707010391). We thank Professor Andrew for helpful discussions.

\section{References}

1 G. Song and Q. Du, J. Chromatogr. A, 2010, 1217, 5930-5934.

2 J. K. Yan, H. L. Ma, J. J. Pei, Z. B. Wang and J. Y. Wu, Sep. Purif. Technol., 2014, 135, 278-284.

3 I. Giavasis, Curr. Opin. Biotechnol., 2014, 26, 162-173.

4 A. Staub, Methods Carbohydr. Chem., 1965, 5, 5-6.

5 R. Yang, D. Meng, Y. Song, J. Li, Y. Zhang, X. Hu, Y. Ni and Q. Li, J. Agric. Food Chem., 2012, 60, 8450-8456.

6 S. Kothari, J. A. Kim, N. Kothari, C. Jones, W. S. Choe and R. Carbis, Vaccine, 2014, 32, 2457-2462.

7 H. S. G. Tan, B. Yu, P. Curran and S. Q. Liu, Food Chem., 2011, 124, 80-84.

8 Y. Lu, H. Yao, C. Li, J. Han, Z. Tan and Y. Yan, Food Chem., 2016, 192, 163-170.

9 A. Greve and M.-R. Kula, Bioprocess Eng., 1991, 6, 173-177. 10 Z. G. Li, H. Teng and Z. L. Xiu, Process Biochem., 2010, 45, 731-737.

11 Z. G. Li, B. Jiang, D. J. Zhang and Z. L. Xiu, Sep. Purif. Technol., 2009, 66, 472-478.

12 Z. G. Li, H. Teng and Z. L. Xiu, Process Biochem., 2011, 46, 586-591.

13 Z. G. Li, Y. Q. Sun, W. L. Zheng, H. Teng and Z. L. Xiu, Biochem. Eng. J., 2013, 80, 68-75.

14 J. K. Yan, W. Q. Wang and J. Y. Wu, J. Funct. Foods, 2014, 6, 33-47.

15 R. K. Joshi, J Med Plants, 2016, 4, 65-67.

16 Y. X. Gong, S. P. Li, P. Li, J. J. Liu and Y. T. Wang, J. Chromatogr. A, 2004, 1055, 215-221.
17 H. Ni, X. H. Zhou, H. H. Li and W. F. Huang, J. Chromatogr. B: Anal. Technol. Biomed. Life Sci., 2009, 877, 2135-2141.

18 P. X. Chen, S. Wang, S. Nie and M. Marcone, J. Funct. Foods, 2013, 5, 550-569.

19 M. G. Shashidhar, P. Giridhar, K. U. Sankar and B. Manohar, J. Funct. Foods, 2013, 5, 1013-1030.

20 H. Miller, Int. Fiber J., 2000, 15, 14-16.

21 L. Deng, T. Y. Zhou, L. Pi, X. H. Zhao, T. Han, Y. K. Li and F. Han, Asian J. Chem., 2013, 25, 1-7.

22 L. f. Huang, M. j. Wu, X. j. Sun, F. q. Guo, Y. Z. Liang and X. R. Li, J. Cent. South Univ. Technol., 2004, 11, 295-299.

23 J. Y. Ling, Y. J. Sun, H. Zhang, P. Lv and C. K. Zhang, J. Biosci. Bioeng., 2002, 94, 371-374.

24 M. Dubois, K. A. Gilles, J. K. Hamilton, P. Rebers and F. Smith, Anal. Chem., 1956, 28, 350-356.

25 D. M. Lan, N. Yang, W. K. Wang, Y. F. Shen, B. Yang and Y. H. Wang, Int. J. Mol. Sci., 2011, 12, 3950-3965.

26 Z. L. Xiu, W. Jiang and Z. G. Su, J. Chem. Ind. Eng., 1993, 6, 018.

27 B. J. Wang, S. J. Won, Z. R. Yu and C. L. Su, Food Chem. Toxicol., 2005, 43, 543-552.

28 J. Y. Ling, G. Y. Zhang, J. Q. Lin, Z. J. Cui and C. K. Zhang, Sep. Purif. Technol., 2009, 66, 625-629.

29 Y. Wang, H. Zhao, X. Miao, D. Liu, H. Jiang, P. Liu, Y. Wang and H. Yin, Fitoterapia, 2013, 86, 13-18.

30 W. Fan, K. Shang, G. Shan and P. Pan, RSC Adv., 2016, 6, 59352-59359.

31 P. K. Grover and R. L. Ryall, Chem. Rev., 2005, 105, 1-10.

32 L. Zhang, Y. Wang and S. Wu, RSC Adv., 2017, 7, 1159111600.

33 Y. Chen, M. Y. Xie, S. P. Nie, C. Li and Y. X. Wang, Food Chem., 2008, 107, 231-241. 